\title{
Comparison of Disk Diffusion and E-Test with the Reference Method of Microbroth Dilution for Susceptibility Testing of Acinetobacter baumannii Isolates to Tetracyclines
}

\author{
Abdollah Ardebili (PhD) \\ Department of Microbiology, \\ Faculty of Medicine, Golestan \\ University of Medical Sciences, \\ Gorgan, Iran
}

Malihe Talebi (PhD)

Department of Microbiology, Faculty of Medicine, Iran

University of Medical Sciences, Tehran, Iran

Abdolaziz Rastegar Lari (PhD)

Department of Microbiology, Faculty of Medicine, Iran

University of Medical Sciences,

Tehran, Iran

Corresponding Author:

Abdolaziz Rastegar Lari

Tel: $+98(21) 86703183$

E-mail: azizlari@gmail.com

Address: Professor of Medical Bacteriology, Department of Microbiology, Faculty of Medicine, Iran University of Medical Sciences, Tehran, Iran

Received : 03 jun 2014

Final edit: 30 aug 2014

Accepted: 08 sep 2014

\begin{abstract}
Background and Objective: Due to the continuous increase of multidrug-resistant Acinetobacter baumannii strains around the world, decision making for an effective treatment of infections caused by this organism depends on the results of antimicrobial susceptibility tests. In this study, the validity of disk diffusion and E-test methods was assessed by their comparison with the reference method of microbroth dilution for three antibiotics of tetracycline, doxycycline and minocycline.

Methods: Total of $68 \mathrm{~A}$. baumannii isolates were obtained from patients hospitalized in the burn center of Shahid Motahari Hospital in Tehran, Iran. Susceptibility of the Acinetobacter isolates was evaluated using the disk diffusion, E-test and microbroth dilution methods, according to the guidelines of Clinical and Laboratory Standards Institute.

Results: Among the isolates, $82.3 \%$ were tetracycline-resistant (with minimum inhibitory concentration 50 (IIC ${ }_{50}$ ) and $\mathrm{MIC}_{90}$ of 32 and more than $32 \mu \mathrm{g} / \mathrm{ml}$, respectively and 41.2\% were doxycycline-resistant (with $\mathrm{VIC}_{50}$ and $\mathrm{MIC}_{90}$ of 4 and more than $32 \mu \mathrm{g} / \mathrm{ml}$, respectively). Minocycline, with resistance of up to $13.3 \%$ ( $\mathrm{NIC}_{50}$ and $\mathrm{MIC}_{90}$ of 1 and $8 \mu \mathrm{g} / \mathrm{ml}$, respectively) showed the highest antimicrobial activity against the $A$. baumannii isolates. Antimicrobial susceptibility of bacteria was different depending on the type of methods used. No very major error was observed in any of the methods of susceptibility testing. 0verall, the level of major and minor errors in the E-test was lower than the disk diffusion method.

Conclusion: The results of this study indicate that minocycline has notably high antimicrobial activity against $A$. baumannii compared to other antibiotics of the tetracycline group.

Keywords: Acinetobacter baumannii; Tetracyclines; Minimum inhibitory concentration.
\end{abstract}




\section{INTRODUCTION}

Acinetobacter baumannii (A. baumannii) is an obligate aerobic, gram-negative coccobacillus which has recently become an important nosocomial pathogen, especially in intensive care units (ICUs) and burn centers $(1,2,3)$. Most infections caused by this organism occur in people with underlying diseases and include pneumonia, urinary tract infection, burn wound infection, meningitis, endocarditis, and septicemia $(4,5,6)$. Regarding the clinical importance, $A$. baumannii is considered the second most common non-fermentative bacteria associated with nosocomial infections. It is also responsible for $10 \%$ to $43 \%$ of mortalities in hospitalized patients in ICU (7).

Tetracyclines are bacteriostatic agents that prevent protein synthesis by inhibiting the binding of aminoacyl-tRNA complex to the ribosome binding site. Although the use of tetracyclines for the treatment of human infections has decreased due to emergence of bacterial resistance, this group of antibiotics, particularly doxycycline and minocycline are still useful antimicrobials against many bacterial infections, including those caused by the Acinetobacter species $(8,9)$.

One of the notable features of $A$. baumannii is its intrinsic or acquired resistance to various antibiotics, including aminopenicillins, cephalosporins, cephamycins, aminoglycosides, tetracyclines and fluoroquinolones. Until recently, the carbapenems (imipenem) was considered as highly active antibacterial agent against $A$. baumannii infections. However, nowadays various reports of increased number of carbapenem resistant strains are found worldwide (10). Since the fact that multi-drug resistance among A. baumannii strains is continually increasing, the final decision for effective treatment of infections caused by this bacterium in hospitalized patients depends on the results of susceptibility tests $(11,12)$.

The aim of this study was to compare disk agar diffusion and E-test methods with the microbroth dilution reference method for susceptibility testing of $A$. baumannii isolates to three tetracyclines, including tetracycline, doxycycline, and minocycline.

\section{MATERIAL AND METHODS}

A total of 68 A. baumannii isolates were recovered from burn patients hospitalized in Shahid Motahari Medical and Rehabilitation Center in Tehran, Iran. Bacterial isolates were obtained from patients aged from 2 to 75 years with burn severity of $7 \%$ to $92 \%$, who were hospitalized in the medical center for at least one week. Burn wound exudate samples were collected and then transported to the laboratory for microbiological examinations. Initial identification of $A$. baumannii isolates was done by traditional bacteriological and biochemical tests such as, Gram staining, catalase, oxidase, motility, OxidationFermentation (OF), and growth at temperatures of 37 and $44{ }^{\circ} \mathrm{C}(13)$.

Polymerase chain reaction (PCR) assay was used as a species confirmatory test to detect the carbapenemase $b l a_{\text {Oxa-51-like gene, which is }}$ intrinsically present in all $A$. baumannii strains (14). Briefly, bacterial genomic DNA was extracted using DNA Purification Kit (Fermentase, Germany). Spectrophotometry was used to evaluate the quality of the DNA by measuring the concentration and relative absorbance of A260/280 for each isolate. DNA in each microtube was kept at $-80{ }^{\circ} \mathrm{C}$ until the PCR assay. The specific primer pairs used for gene amplification are 5'TAATGCTTTGATCGGCCTTG-3' and 5'TGGATTGCACTTCATCTTGG-3'. PCR was done according to the following procedure using the Mastercycler gradient (Eppendorf, Germany): Initial denaturation at $94{ }^{\circ} \mathrm{C}$ for 5 minutes and then 30 cycles of denaturation at $94{ }^{\circ} \mathrm{C}$ for 45 seconds, annealing at $58{ }^{\circ} \mathrm{C}$ for 1 minute, extension at $72{ }^{\circ} \mathrm{C}$ for 1 minute and final extension at $72{ }^{\circ} \mathrm{C}$ for 5 minutes. PCR products were examined for the presence of DNA bands using a UV transilluminator, after electrophoresis on ethidium bromidecontaining $1.5 \%$ agarose gel.

Susceptibility of all A. baumannii isolates to tetracycline, doxycycline, and minocycline was evaluated using disk agar diffusion $(30 \mu \mathrm{g}$ for each antibiotics) (Mast Diagnostics, Merseyside, UK), microbroth dilution (Becton Dickinson, Sparks, MD, USA) and E-test 
methods (AB Biodis Solna, Sweden Merseyside, UK), according to the guidelines of Clinical \& Laboratory Standards Institute (CLSI) (15,16). Standard strains of Escherichia coli (ATCC 25922) and Pseudomonas aeruginosa (ATCC 27852) were used as quality controls. Serial dilutions of antibiotics were prepared according to the manufacturer's instruction in the microbroth dilution method: $0.25-32 \mu \mathrm{g} / \mathrm{ml}$ for tetracycline and minocycline, and 0.125-32 $\mu \mathrm{g} / \mathrm{ml}$ for doxycycline. Interpretive standards related to MIC of all three antibiotics in two microbroth dilution and E-test methods were as follows: susceptible; MIC $\leq 4 \mu \mathrm{g} / \mathrm{ml}$, intermediate; $\mathrm{MIC}=8 \mu \mathrm{g} / \mathrm{ml}$, and resistant; MIC $\geq 16 \mu \mathrm{g} / \mathrm{ml}$. The results of antimicrobial susceptibility were evaluated and recorded after 20 hours of incubation at $35^{\circ} \mathrm{C}$, according to the CLSI guidelines. Errors related to determination of the antimicrobial susceptibility were categorized as follows: very major error; if the results of the reference microbroth dilution method were resistant, but the results of disk diffusion and E-test were susceptible (false susceptible). Major error; if the results of reference method were susceptible and the results of disk diffusion and E-test were resistant (false resistant). Minor error; if the results of the reference method were susceptible or resistant, but the results of the other two tests were recorded as intermediate.

Data were entered in the SPSS software (Version11.5; SPSS, Inc., Chicago, IL, USA) and Chi-square test was used to analyze the data. P-value of $\leq 0.05$ was considered as statistically significant.

Table 1-MIC distribution of three tetracyclines against $68 \mathrm{~A}$. baumannii isolates

\begin{tabular}{|c|c|c|c|c|c|c|c|c|c|c|c|c|c|}
\hline \multirow[t]{2}{*}{ Antibiotic } & \multirow{2}{*}{$\begin{array}{c}\text { MIC } \\
\text { range } \\
(\mu \mathrm{g} / \mathrm{mL})\end{array}$} & \multicolumn{10}{|c|}{ No. $(\%)$ of isolates with determined MIC $(\mu \mathrm{g} / \mathrm{mL})$} & \multirow{2}{*}{$\begin{array}{c}\mathrm{MIC}_{50} \\
(\mu \mathrm{g} / \mathrm{mL})\end{array}$} & \multirow{2}{*}{$\begin{array}{c}\mathrm{MIC}_{90} \\
(\mu \mathrm{g} / \mathrm{mL})\end{array}$} \\
\hline & & 0.125 & 0.25 & 0.5 & 1 & 2 & 4 & 8 & 16 & 32 & $>\mathbf{3 2}$ & & \\
\hline Tetracycline & $1-\geq 32$ & - & - & - & $1(1.4)$ & $2(2.9)$ & $5(7.3)$ & $4(5.8)$ & $8(11.7)$ & $15(22)$ & $33(48.5)$ & 32 & $>32$ \\
\hline Doxycycline & $0.125-\geq 32$ & $5(7.3)$ & $8(11.7)$ & $6(8.8)$ & $10(14.7)$ & $4(5.8)$ & $2(2.9)$ & $5(7.3)$ & $9(13.2)$ & 9 (13.2) & $10(14.7)$ & 4 & $>32$ \\
\hline Minocycline & 0.25- 32 & - & $6(8.8)$ & $12(17.6)$ & 22 & 12 & $7(10.3)$ & $6(8.8)$ & $2(2.9)$ & $1(1.4)$ & - & 1 & 8 \\
\hline
\end{tabular}

Abbreviations: MIC, Minimum inhibitory concentration.

Table 2- Susceptibility patterns of $\boldsymbol{A}$. baumannii isolates to tetracyclines based on three methods

\begin{tabular}{cccc}
\hline Antibiotic & \multicolumn{3}{c}{ No. $(\%)$ of isolates } \\
& Susceptible & Intermediate & Resistant \\
\hline Tetracycline & & & \\
DAD & $2(2.9)$ & $12(17.6)$ & $54(79.4)$ \\
ET & $4(5.9)$ & $9(13.2)$ & $55(80.9)$ \\
MBD & $8(11.8)$ & $4(5.9)$ & $56(82.3)$ \\
Doxycycline & & & \\
DAD & $27(39.7)$ & $12(17.6)$ & $29(42.6)$ \\
ET & $30(44.1)$ & $14(58.8)$ & $24(35.3)$ \\
MBD & $35(51.5)$ & $5(7.3)$ & $28(41.2)$ \\
Minocycline & & & \\
DAD & $23(33.8)$ & $40(58.8)$ & $5(7.3)$ \\
ET & $47(69.1)$ & $17(25)$ & $4(5.9)$ \\
MBD & $59(86.7)$ & $6(8.8)$ & $3(4.4)$ \\
\hline
\end{tabular}

Abbreviations: ET, E-test; DAD, Disk agar diffusion; BMD, Microbroth dilution. 
Table 3- Rates of susceptibility testing errors among $68 \mathrm{~A}$. baumannii isolates by antimicrobial agent and testing method

\begin{tabular}{|c|c|c|c|c|}
\hline \multirow{2}{*}{$\begin{array}{l}\text { Antibiotic and } \\
\text { testing method }\end{array}$} & \multirow{2}{*}{$\begin{array}{c}\% \\
\text { susceptible } \\
\text { isolates }\end{array}$} & \multicolumn{3}{|c|}{$\%^{a}$} \\
\hline & & Very major error $^{b}$ & Major error $^{c}$ & Minor error ${ }^{d}$ \\
\hline \multicolumn{5}{|l|}{ Tetracycline } \\
\hline DAD & 2.9 & 0 & 11.7 & 1.4 \\
\hline ET & 5.9 & $\mathbf{0}$ & 8.8 & 1.4 \\
\hline MBD & 11.8 & & & \\
\hline \multicolumn{5}{|l|}{ Doxycycline } \\
\hline DAD & 39.7 & 0 & 5.9 & 17.6 \\
\hline ET & 44.1 & 0 & 4.4 & 10.3 \\
\hline MBD & 51.5 & & & \\
\hline \multicolumn{5}{|l|}{ Minocycline } \\
\hline DAD & 33.8 & 0 & 2.9 & 50 \\
\hline ET & 69.1 & $\mathbf{0}$ & 1.4 & 16.2 \\
\hline MBD & 86.7 & & & \\
\hline
\end{tabular}

\footnotetext{
Abbreviations: ET, E-test; DAD, Disk agar diffusion; BMD, Microbroth dilution.

${ }^{a}$ Unacceptable levels are shown in bold. According to CLSI, they are $\geq 1.5 \%, \geq 3 \%$, and $\geq 10 \%$ for very major error, major error, and minor error as, respectively.

${ }^{b}$ Very major error, susceptible by DAD or ET when resistant by MBD.

${ }^{c}$ Major error, resistant by DAD or ET when susceptible by MBD.

${ }^{\text {d }}$ Minor error, intermediate by DAD or ET when susceptible or resistant by MBD or susceptible or resistant by

DAD or ET when intermediate by MBD.
}

\section{RESULTS}

All 68 bacterial isolates that were identified by routine microbiological and biochemical methods harbored the $b l a_{\text {Oxa-51- }}$ like gene, confirming the A. baumannii species. $b{ }_{\text {Oxa-51 }}$-like gene amplification in the clinical isolates and A. baumannii standard strain (positive control) produced a $353 \mathrm{bp}$ band, while no amplification was observed in A. lwoffii strain (negative control).

Among the studied antibiotics, minocycline had the highest antimicrobial activity based on the findings of microbroth dilution method $(86.7 \%$ of isolates were susceptible with $\mathrm{MIC}_{50}$ and $\mathrm{MIC}_{90}$ values of 1 and $8 \mu \mathrm{g} / \mathrm{ml}$, respectively). Also, $82.3 \%$ and $41.2 \%$ of the isolates showed full resistance (MIC > $16 \mu \mathrm{g} / \mathrm{ml}$ ) to tetracycline and doxycycline, respectively. Moreover, bacterial isolates had $\mathrm{MIC}_{50}$ and $\mathrm{MIC}_{90}$ of 32 and $>32 \mu \mathrm{g} / \mathrm{ml}$ versus 4 and $>32 \mu \mathrm{g} / \mathrm{ml}$ to tetracycline and doxycycline, respectively (Tables 1 and 2).

The accuracy of the disk diffusion and E-test methods was evaluated by comparison of results of 68 Acinetobacter isolates tested by these methods to the reference microbroth dilution (Table 3). Susceptibility of isolates to minocycline was varied based on the methods used, ranging from $33.8 \%$ by disk diffusion to $86.7 \%$ by the microbroth dilution method. There was no very major error in any methods of susceptibility testing. The E-test showed fewer major errors for all three antibiotics $(8.8 \%, 4.4 \%$ and $4.1 \%$ major errors for tetracycline, doxycycline and minocycline, respectively), when compared with the disk diffusion method. In addition, minor error was equivalent for tetracycline in E-test and disk diffusion methods $(1.4 \%)$; while it was fewer for minocycline and doxycycline in the E-test (10.3\% and $16.2 \%$, respectively) (Table 3 ).

In the present study, the level of major error for minocycline in the disk diffusion and Etest methods were acceptable; whereas it was unacceptable for tetracycline and doxycycline. In contrast to our study, Swenson et al. reported high level of minor errors $(32.1 \%)$ by disk diffusion for tetracycline. However, the major errors were the same and, of course, unacceptable according to the CLSI in both studies.

\section{DISCUSSION}

Currently, A. baumannii, like other members of the "ESCAPE" group (Enterococcus faecium, Staphylococcus aureus, Klebsiella pneumoniae, Acinetobacter baumannii, Pseudomonas aeruginosa and Enterobacter species) has become a serious challenge in controlling and treatment of infections $(17,18)$. This forces physicians and infectious specialists to use a broad-spectrum antibiotics for treatment, which in turn provides selective pressure allowing the organism to acquire various resistance genes $(19,20)$. Therefore, control and treatment of infections caused by this organism is clearly dependent on laboratory tests that identify or 
evaluate antimicrobial resistance. Several methods are used in clinical microbiology laboratories to evaluate the antimicrobial susceptibility of microorganisms. In the present study, the accuracy of disk diffusion and E-test results for three tetracyclines was assessed by comparing to the reference microbroth dilution method. Overall, the accuracy of disk diffusion test for determining minocycline susceptibility was low; so that $52.9 \%$ of the isolates were incorrectly considered as nonsusceptible (50\% and $2.9 \%$ as intermediate- and full resistant, respectively). This likely occurred owing to clustering of the inhibition zone diameter at or near the susceptibility breakpoint for minocycline in many A. baumannii strains. By the E-test, $17.6 \%$ of isolates were incorrectly reported as minocycline-nonsusceptible $(1.4 \%$ and $16.2 \%$ as intermediate- and full-resistant, respectively), a significant difference and substantial improvement in accurate results over the disk diffusion. Findings obtained from our study is consistent with those of Akers et al. in the United States (21), in which $51 \%$ versus $18 \%$ of $A$. baumannii isolates were wrongly reported as minocycline nonsusceptible by disk diffusion and E-test methods, respectively.

In agreement with others (21), our study indicates that minocycline has adequate antimicrobial activity against $A$. baumannii.

\section{REFERENCES}

1. Humphreys H, Towner KJ. Impact of Acinetobacter spp. in intensive care units in Great Britain and Ireland. J Hosp Infect. 1997; 37(4): 281-286.

2. Ardebili A, Talebi M, Azimi L, Rastegar Lari A. Effect of efflux pump inhibitor CCCP on the MIC of ciprofloxacin in Acinetobacter baumannii clinical isolates. Jundishapur J Microbio. 2014; 7(1): doi:10.5812/jjm.8691.

3. Sharma BR. Infection in patients with severe burns: causes and prevention thereof. Infect Dis Clin North Am. 2007; 21(3): 745-759.

4- Chastre J. Infections due to Acinetobacter baumannii in the ICU. Semin Respir Crit Care Med. 2003; 24(1): 69-78.

5. Munoz-Price LS, Weinstein RA. Acinetobacter infection. N Engl J Med. 2008; 358: 1271-1281.

6. Murray CK, Hospenthal DR. Treatment of multidrug resistant Acinetobacter. Curr Opin Infect Dis. 2005; 18(6): 502-506.

7. Falagas ME, Rafailidis PI. Attributable mortality of Acinetobacter baumannii: no longer a controversial issue. Crit Care. 2007; 11(3): 134. doi:10.1186/cc5911.
Similarly, in the Maleki et al.'s study (22), only $11.8 \%$ of isolates were susceptible to tetracycline and high level of resistance was reported to this antibiotic ( $\mathrm{MIC}_{50}$ and $\mathrm{MIC}_{90}$ of 32 and $\geq 32 \mu \mathrm{g} / \mathrm{ml}$, respectively). On the other hand, only $19 \%$ of the isolates exhibited resistance to minocycline. A key point regarding the assessing the susceptibility of isolates to minocycline is the important difference in accuracy between susceptibility testing methods, as significantly high level of false resistance are observed by the disk agar diffusion. Therefore, the use of the reference microbroth dilution method is recommended to obtain the most accurate susceptibility determination in the cases of $A$. baumannii infections where minocycline is desirable.

\section{CONCLUSION}

In order to determine the trend of antimicrobial susceptibility of multidrug resistant (MDR) A. baumannii strains, and subsequently to choose an efficient antibiotic regimen for treatment of problematic infections, it is recommended to use the accurate susceptibility testing methods.

\section{ACKNOWLEDGMENT}

The authors would like to thank the Iran University of Medical Sciences for the financial support of this research project (code 1067).

\section{CONFLICTS OF INTEREST}

Therer are no conflicts of interest.

8. Huys G, Cnockaert M, Vaneechoutte M, Woodford N, Nemec A, Dijkshoorn L, et al. Distribution of tetracycline resistance genes in genotypically related and unrelated multiresistant Acinetobacter baumannii strains from different European hospitals. Res Microbiol. 2005; 156(3): 348-355.

9. Jones RN, Ferraro MJ, Reller LB, Schreckenberger PC, Swenson JM, Sader HS. Multicenter studies of tigecycline disk diffusion susceptibility results for Acinetobacter spp. J Clin Microbiol. 2007; 45(1): 227230.

10. Huang L, Sun L, Xu G, Xia T. Differential susceptibility to carbapenems due to the AdeABC efflux pump among nosocomial outbreak isolates of Acinetobacter baumannii in a Chinese hospital. Diagn Microbiol Infect Dis. 2008; 62(3): 326-32. doi: 10.1016/j.diagmicrobio.2008.06.008.

11. Dent LL, Marshall DR, Pratap S, Hulette RB. Multidrug resistant Acinetobacter baumannii: a descriptive study in a city hospital. BMC Infect Dis. 2010; 10: 196. doi: 10.1186/1471-2334-10-196. 
12. Swenson JM, Killgore GE, Tenover FC. Antimicrobial susceptibility testing of Acinetobacter spp. by NCCLS broth microdilution and disk diffusion methods. J Clin Microbiol. 2004; 24(11): 5102-5108.

13. Koneman EW, Allen SD, Janda WM, Schreckenberger PC, Winn Jr WC. The non-fermentative Gram-negative bacilli. In: Color atlas and textbook of diagnostic microbiology. 6 th ed. Philadelphia: Lippincott-Williams \& Wilkins Publishers. 2006; 304391.

14. Turton JF, Woodford N, Glover J, Yarde S, Kaufmann ME, Pitt TL. Identification of Acinetobacter baumannii by detection of the blaOXA-51-like carbapenemase gene intrinsic to this species. J Clin Microbiol. 2006; 44(8): 2974-2976.

15. CLSI. Performance standards for antimicrobial susceptibility testing. Twenty-third informational supplement: Wayne, PA: 2013; M100-S23.

16. CLSI. Methods for dilution antimicrobial susceptibility tests for bacteria that grow aerobically; approved standard, 8th ed. Wayne, PA: 2009; M7-A8.

17. Boucher HW, Talbot GH, Bradley JS, Edwards JE, Gilbert D, Rice LB, et al. Bad bugs, no drugs: no ESKAPE! An update from the Infectious Diseases Society of America. Clin Infect Dis. 2009; 48(1): 1-12. doi: $10.1086 / 595011$.
18. Ardebili A, Rastegar Lari A, Talebi M. Correlation of ciprofloxacin resistance with the AdeABC efflux system in Acinetobacter baumannii clinical isolates. Ann Lab Med. 2014;34:433-438.

19. Rice LB. Federal funding for the study of antimicrobial resistance in nosocomial pathogens: no ESKAPE. J Infect Dis. 2008; 197(8): 1079-1081. doi: $10.1086 / 533452$.

20. Valentine SC, Contreras D, Tan S, Real LJ, Chu S, $\mathrm{Xu} \mathrm{HH}$. Phenotypic and molecular characterization of Acinetobacter baumannii clinical isolates from nosocomial outbreaks in Los Angeles County, California. J Clin Microbiol. 2008; 46(8): 2499-2507. doi: 10.1128/JCM.00367-08.

21. Akers KS, Mende K, Yun HC, Hospenthal DR, Beckius ML, Yu X, et al. Tetracycline susceptibility testing and resistance genes in isolates of Acinetobacter baumannii-Acinetobacter calcoaceticus complex from a U.S. military hospital. Antimicrob Agents Chemother. 2009; 53(6): 2693-2695.

22. Maleki MH, Sekawi Z, Soroush S, Azizi-Jalilian F, Asadollahi Kh, Mohammadi S, et al. Phenotypic and genotypic characteristics of tetracycline resistant Acinetobacter baumannii isolates from nosocomial infections at Tehran hospitals. Iran J Basic Med Sci. 2014; 17(1): 21-26. 